\title{
Fusion noise-removal technique with modified dark-contrast algorithm for robust segmentation of acute leukemia cell images
}

\author{
Nor Hazlyna Harun a,1,*, Juhaida Abu Bakar a,2, Hamirul Aini' Hambali a,3, Nurnadia Mohd Khair ${ }^{\text {b,4, }}$, \\ Mohd. Yusoff Mashor ${ }^{\mathrm{c}, 5}$, Roseline Hassan d,6 \\ a Data Science Research Lab, School of Computing, College of Arts and Science, Universiti Utara Malaysia, Kedah, Malaysia \\ ${ }^{b}$ Faculty of Engineering Technology, Universiti Malaysia Perlis, Perlis, Malaysia \\ c School of Mechatronics Engineering, Universiti Malaysia Perlis, Perlis, Malaysia \\ d Department of Hematology, Universiti Sains Malaysia, Kelantan, Malaysia \\ ${ }^{1}$ hazlyna@uum.edu.my; ${ }^{2}$ juhaida.ab@uum.edu.my; ${ }^{3}$ hamirul@uum.edu.my; ${ }^{4}$ uwais1973@yahoo.com.my; \\ ${ }^{5}$ yusoff@unimap.edu.my; ${ }^{6}$ roslin@usm.my \\ * corresponding author
}

\section{ARTICLE INFO}

\section{Article history}

Received June 4, 2018

Revised July 11, 2018

Accepted August 15, 2018

Available online November 11, 2018

\section{Keywords}

Acute leukemia

Median filter

Seeded region growing area

Extraction

Modified dark contrast enhancement

\section{ABSTRACT}

Segmentation is the major area of interest in the field of image processing stage. In an automatic diagnosis of acute leukemia disease, the crucial process is to achieve the accurate segmentation of acute leukemia blood image. Generally, there are three requirements of image segmentation for medical purposes, namely; accuracy, robustness and effectiveness which have received considerable critical attention. As such, we propose a new (modified) dark contrast enhancement technique to enhance and automatically segment the acute leukemic cells. Subsequently, we used a fusion $7 \times 7$ median filter as well as the seeded region growing area extraction (SRGAE) algorithm to minimise the salt-and-pepper noise, apart from preserving the post-segmentation edge. As per the outcomes, the accuracy, sensitivity, and specificity of this method were $91.02 \%$, $83.68 \%$, and $91.57 \%$ respectively.

This is an open access article under the CC-BY-SA license.

\section{Introduction}

Leukemia is one type of cancer that occur when abnormal white blood cell continue grow and divide in bone marrow. Normally, leukemia disease can make body difficultly to transport oxygen, fight infections and control bleeding. Acute myeloid leukemia (AML), acute lymphocytic leukemia (ALL), chronic myeloid leukemia (CML) and chronic lymphocytic leukemia (CLL) are the four kinds of leukemia. Nowadays, leukemia is one of dangerous disease that can lead to imminent death. A report in 2009 from the National Cancer Institute has estimated that 12,330 people (5,740 women and 6,590 men) have been diagnosed with AML and 5,330 people (2,180 women and 3,150 men) have been diagnosed with ALL in 2010 [1]. In India, approximately 104,239 total number of individuals suffering from leukemia in 2010. Additionally, a survey in 2012 in the United States has shown that 148,040 have been diagnosed, and 54,380 died of leukemia, lymphoma, and myeloma [2]. Based on these statistics, it is important to develop an automated leukemia detection system due to its high consumption in medical diagnostics, especially for cost-effective and fast production of blood cell count reports.

Various of techniques can be employed to perform segmentation process which can be thresholdingbased, region-based [3]-[5], pixel-based [6]-[8], edge-based [9]-[11], clustering based and classification 
based [12]-[14] and layer based or block based [15]. Recently, studies have examined on the provision of thresholding [16], [17], clustering algorithm [18]-[20], active contour [21], [22] and watershed algorithm [23]. These researchers reported that the studies produced an acceptable performance. Despite the complex nature of blood cell images, most acute leukemia images obtained from the diagnostic procedure suffers from number of problems such as blurred, low contrast and provides unwanted noise. These can cause difficulties to interpret the important leukemia morphologies, at the same time increasing false diagnosis. In the literature, contrast is one of the important factors that influenced the accuracy of leukemia cell interpretation which capable to improve the quality of the image [24]-[29]. Hence, this work aims to contribute to this growing area of research by proposing an in-complex algorithm yet fast to perform accurate detection. Both fusion dark contrast algorithm (DCA) along with removal noise technique have produced robustness segmentation of acute leukemia cells and directly enhanced the percentage of recognition rate for acute leukemia cell.

\section{Method}

\subsection{Image Acquisition}

The samples of acute leukemia image were collected from the Hospital (HUSM) Kubang Kerian Kelantan. To analyze the acute leukemia blood slide images was utilized using a Leica microscope at 40 magnifications. The images were captured by Infinity 2 cameras and saved into (.*bitmap) format with $800 \times 600$ resolution. For the normal and abnormal slides, hematologists validated and confirmed the total cell counts as well as their groups in terms of morphology (i.e. color and shape). Total data used in this study are 100 acute leukemia images (type $A M L=50$ images and type $A L L=50$ images). Fig. 1 (a) and (c) respectively show two examples of original AML and ALL leukemia images, while Fig. 1(b) and (d) represents the intensity histogram of each image. In this stage, histogram is important to ease process selecting threshold value for remain object interest (blast cell-acute leukemia cell) and removed others.

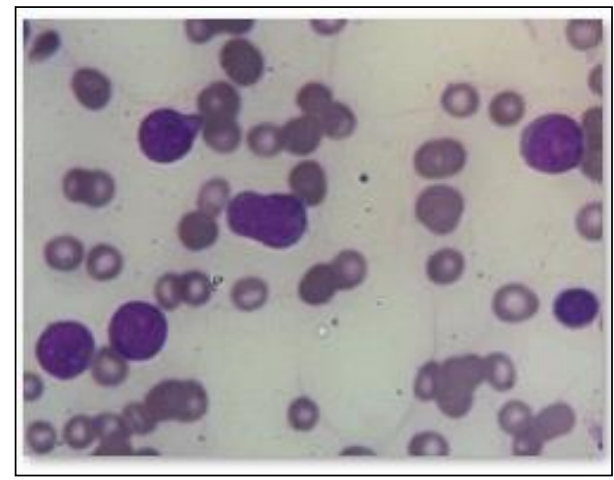

(a) AML image

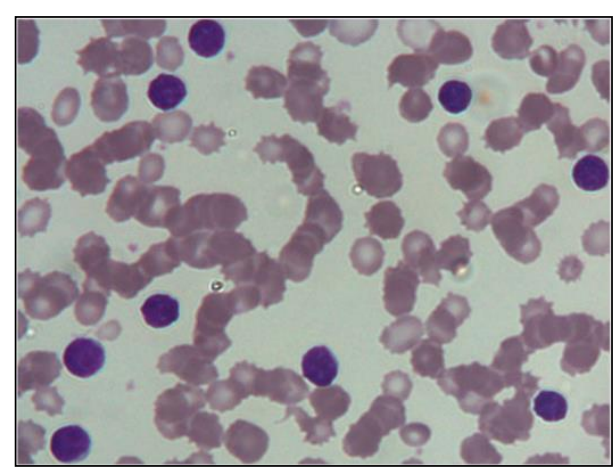

(c) ALL image

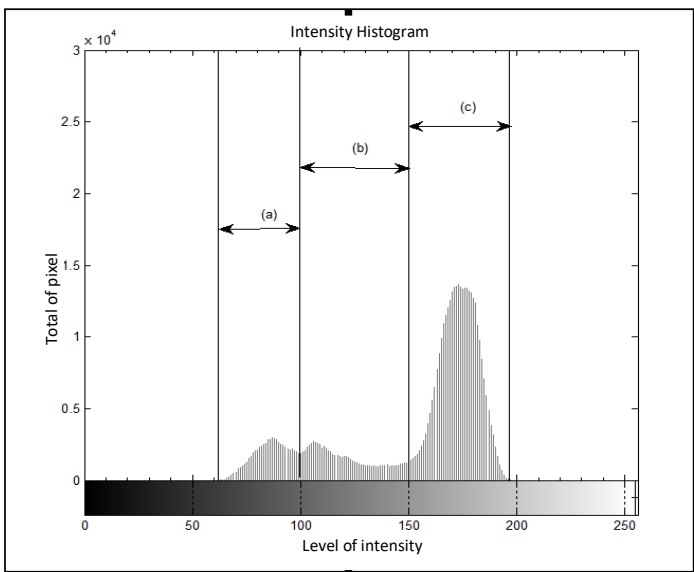

(b) AML histogram

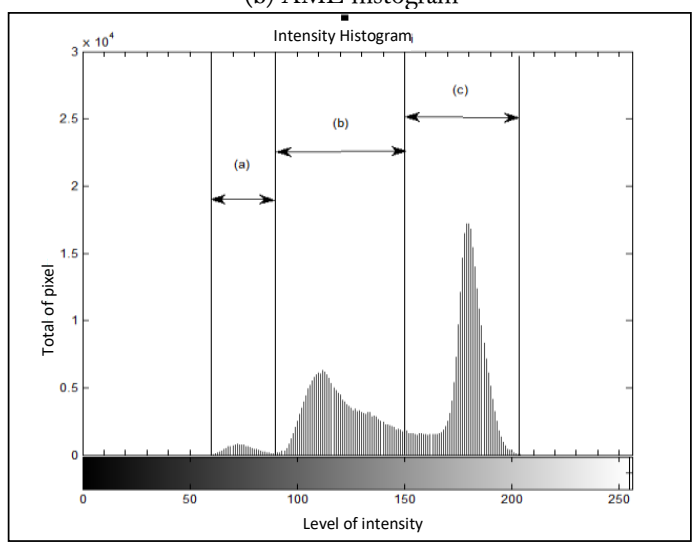

(d) ALL histogram

Fig. 1. Original image $(40 \mathrm{X})$ with intensity histogram 
The shape of the intensity histogram of the four images is almost identical. In other words, the acute blood leukemia image has three major pixel distribution areas such as AML or ALL leukemia cell nucleus, red blood cell and background area. The labels ${ }^{(a)}{ }^{(b)}$ and ${ }^{(c)}$ of each pixel distribution area can be seen in the intensity histogram in Fig. 1(b) and (d) where each represent as a nucleus, red blood cell and background respectively.

\subsection{Proposed Method}

The aims of this proposed method is to provide the clean image by contrast improvement with removing noise, hence producing an effective effect to resultant images for extracting features such as size, color and shape. Firstly, the modification of DCA was applied to improve the image quality and contrast of leukemia image. Fig. 2 illustrates a block diagram of DCA for the purpose of reaching the image of acute leukemia. The dotted line box illustrates the implementation of the DCA methods for enhance the images result before applying the noise removal process. The threshold and strain factor values determined by referring to the histogram of the original image intensity.

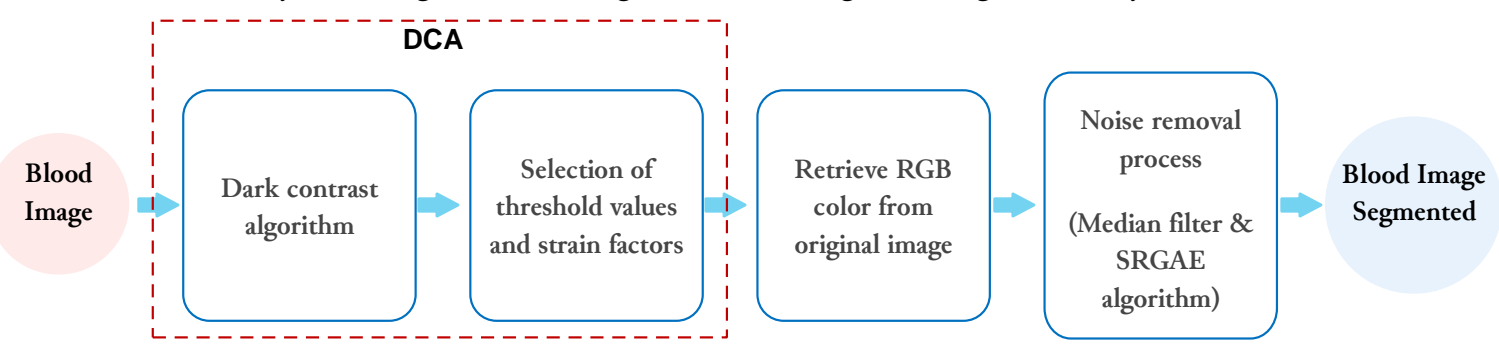

Fig. 2. Block diagram of DCA

\subsubsection{Dark Contrast Method}

In this paper, DCA is used to enhance the image of acute leukemia by increasing the contrast of dark areas in the image. The technique can be defined as (1) [23].

$$
O(x, y)= \begin{cases}\frac{I(x, y)-\min T H}{T H-\min T H} \cdot N T h_{g}, & \text { if } I(x, y)<T H \\ {\left[\frac{I(x, y)-T H}{\max T H-T H} \cdot\left(255-N T h_{g}\right)\right]+N T h_{g},} & \text { if } I(x, y) \geq T H\end{cases}
$$

where $I(x, y)$ is pixels of input image at coordinates $(x, y), O(x, y)$ is pixels of output image at coordinates $(x, y), T H$ is Threshold value and $N T h_{g}$ is Dark stretching factor. The parameters $T H$ and $N T h_{g}$ are threshold values and strain factors respectively while $N T h_{g}$ must be greater than the threshold value.

Fig. 3 illustrates the process of strain and compression pixels using this technique. If the value of the pixel input is less than the threshold value, the pixel will be stretched, otherwise, it will be compressed. The steps as follows: 1) Develop intensity histograms for original AML and ALL blood images; 2) Execute the dark-contrast algorithm on the said images.

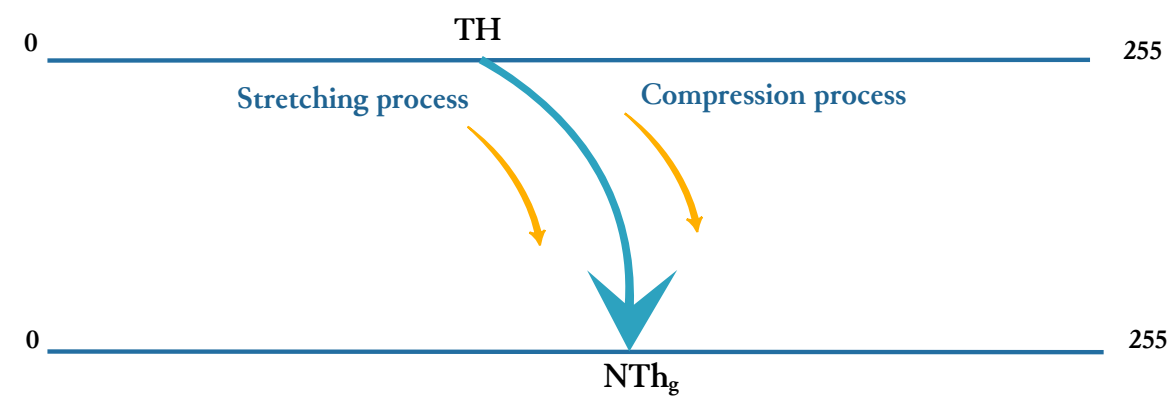

Fig. 3. Dark contrast process 
Choose the cut-off points for $T H$ and $N T H g$ strain factors from the intensity histograms (Fig. 1(b) and (d)) with reference to the areas of pixel distributions of the leukemic cells in area ${ }^{(a)}$; and 3) Retrieve RGB color from the original image using (2).

$$
O(x, y)= \begin{cases}\text { original color } I(x, y), & \text { if } T H \geq \gamma \\ 255, & \text { otherwise } .\end{cases}
$$

where, $O(x, y)$ is the output pixel value and $I(x, y)$ is the original pixel image input value. The recommended value $\gamma$ for $T H$ in this study is 90 . Whereas the value of $N T H_{g}$ dark strain factor is 255 for abnormal blood images by referring to the intensity histogram of the original image (Fig. 1(b) and (d)). TH refer to the threshold value is determined by observation of the intensity histogram for 800 abnormal blood images (AML $=400$ and ALL $=400$ ) that have been recorded during this study process.

Fig. 4 and Fig 5 provided other partials of the sample for acute blood images with the resulted images, and intensity histogram (Fig. 6) for most tested images of type ALL and AML.

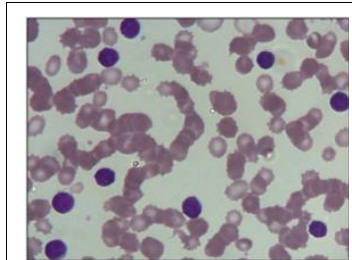

ALL 1

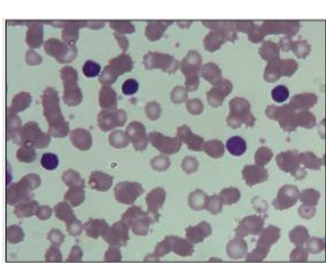

ALL3

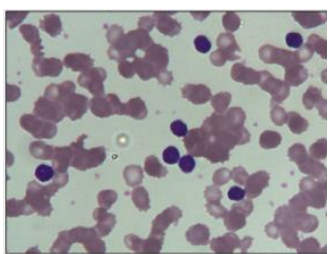

ALL 5

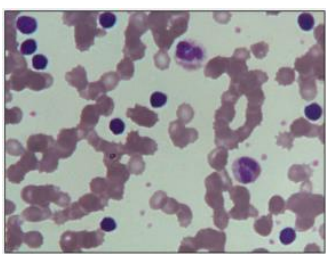

ALL 7

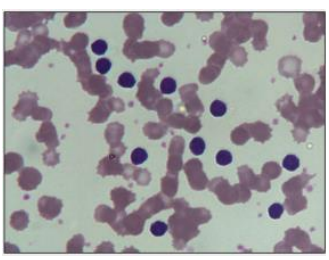

ALL 9

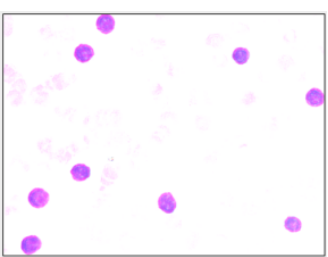

Resulted ALL1

$\left(T H=90, N T H_{g}=255\right)$

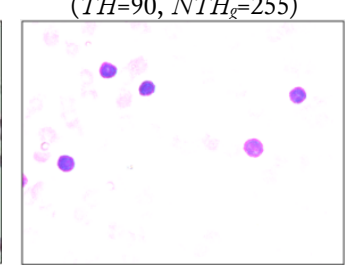

Resulted ALL3 ( $\left.T H=90, N_{T} H_{p}=255\right)$

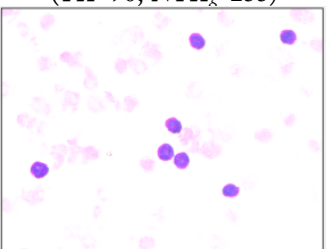

Resulted ALL5
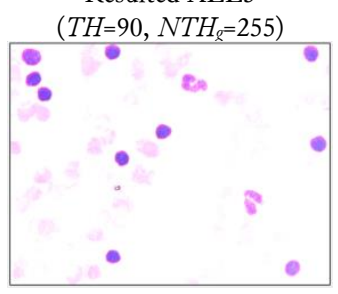

Resulted ALL7 $\left(T H=90, N_{R}=255\right)$

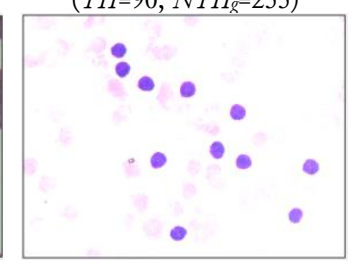

Resulted ALL9 $\left(T H=90, N_{T} H_{g}=255\right)$

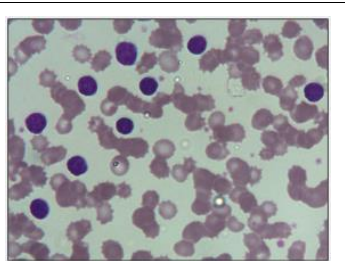

ALL 2

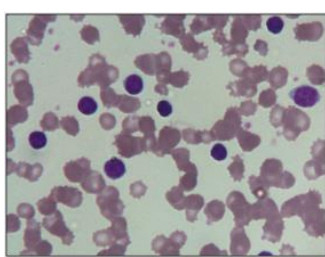

ALL 4

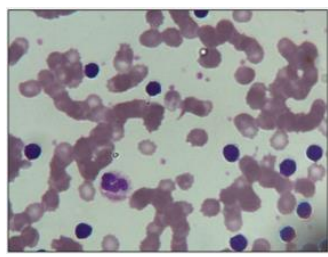

ALL 6

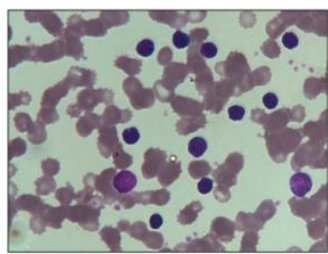

ALL 8

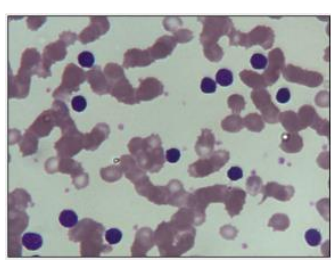

ALL10

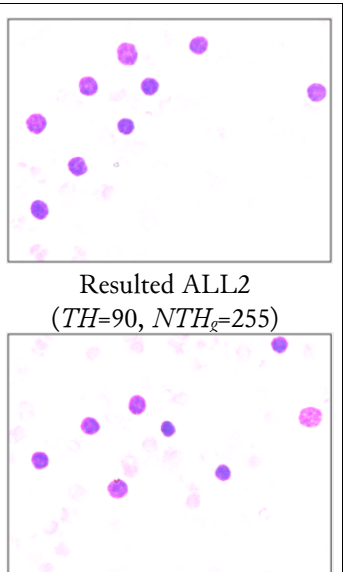

Resulted ALL4 $\left(\mathrm{TH}=90, \mathrm{NTH}_{g}=255\right)$

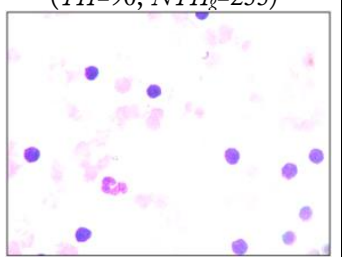

Resulted ALL6 (TH=90, $\left.\mathrm{NTH}_{\mathrm{g}}=255\right)$

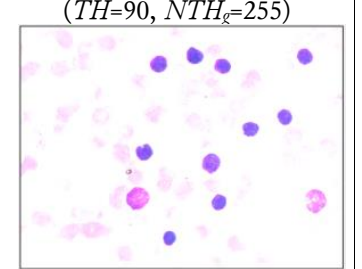

Resulted ALL8 $\left(\mathrm{TH}=90, \mathrm{NTH}_{\mathrm{g}}=255\right)$

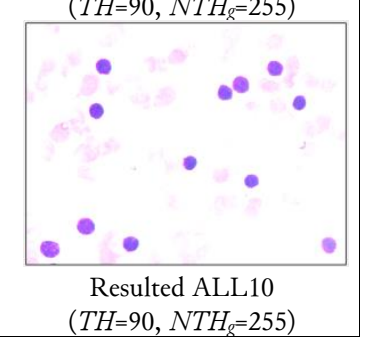

Fig. 4. ALL image 


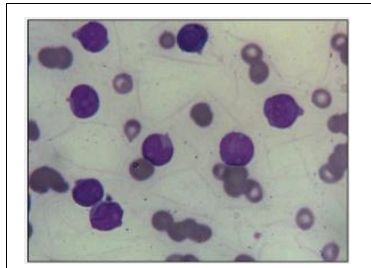

AML 1

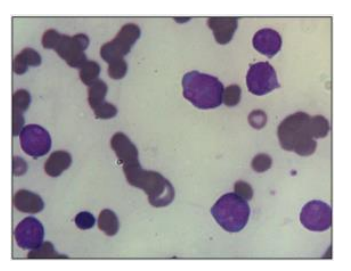

AML 3

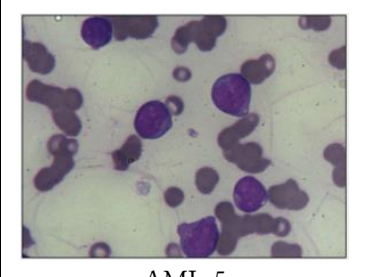

AML 5

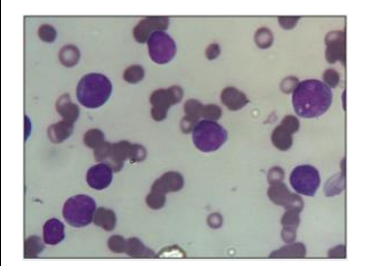

AML 7

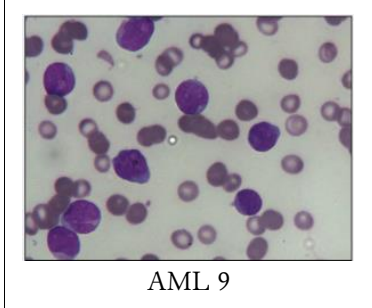

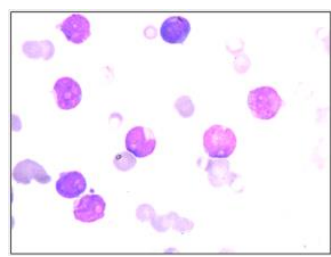

Resulted AML1

$\left(\mathrm{TH}=90, \mathrm{NTH}_{\mathrm{R}}=255\right)$

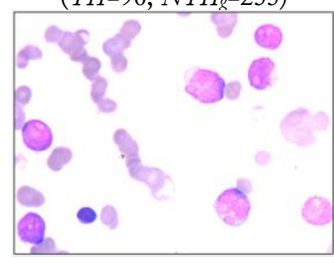

Resulted AML3

$\left(T H=90, N T H_{g}=255\right)$

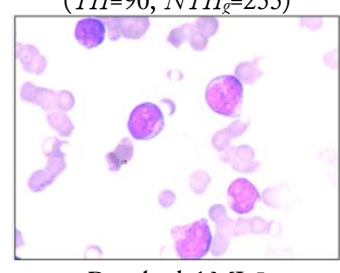

Resulted AML5

$\left(T H=90, N T H_{R}=255\right)$

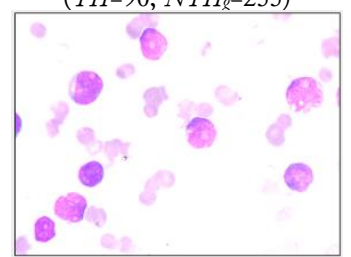

Resulted AML7

$\left(T H=90, N T H_{g}=255\right)$

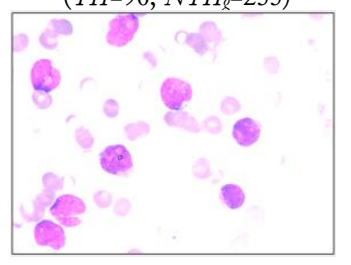

Resulted AML9 $\left(T H=90, N T H_{p}=255\right)$

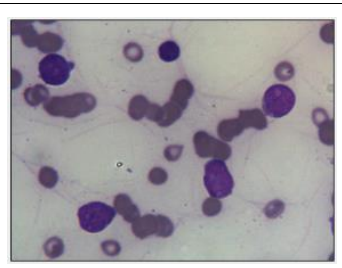

AML 2

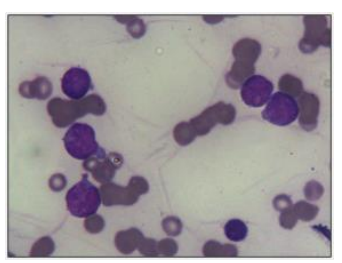

AML 4
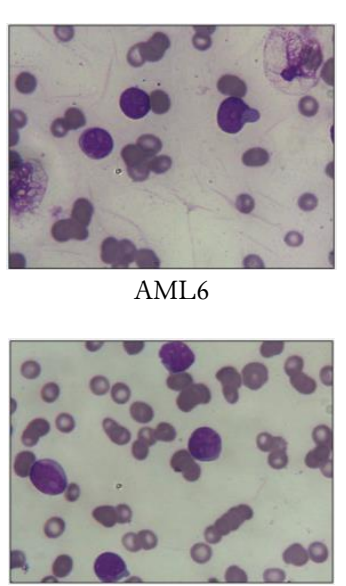

AML 8

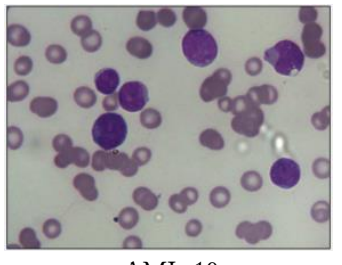

AML 10

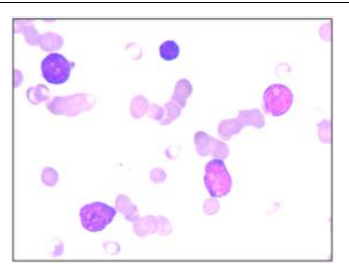

Resulted AML2

$\left(T H=90, N T H_{2}=255\right)$

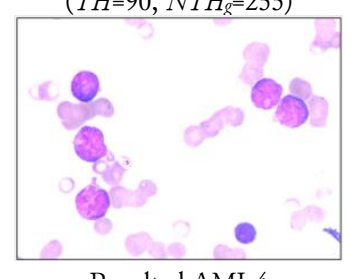

Resulted AML4

$\left(T H=90, N T H_{R}=255\right)$

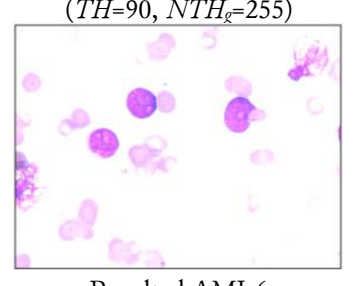

Resulted AML6

$\left(\mathrm{TH}=90, \mathrm{NTH}_{\Omega}=255\right)$

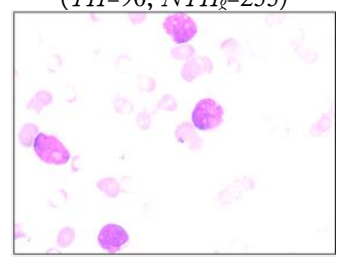

Resulted AML8

$\left(\mathrm{TH}=90, \mathrm{NTH}_{\mathrm{o}}=255\right)$

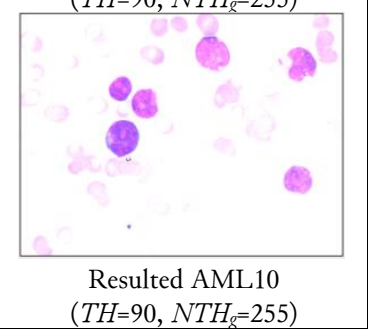

Fig. 5. AML Image
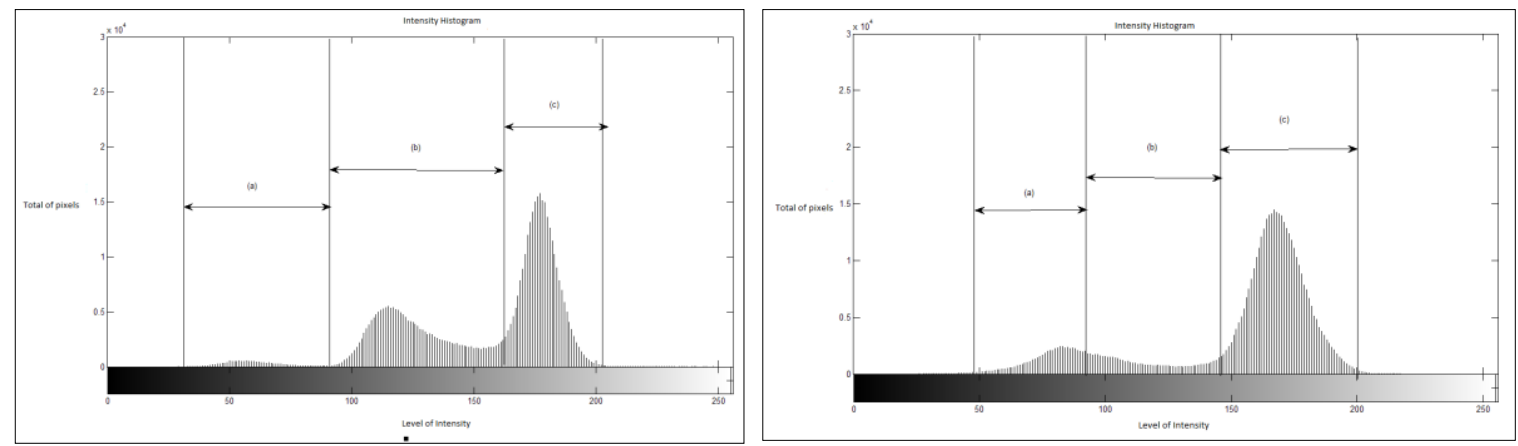

Fig. 6. Intensity histogram (a) ALL Image (b) AML Image 


\subsubsection{Removal noise}

After completing the DCA process, fusion $7 \times 7$ median filter and SRGAE algorithm was used to reduce the salt and pepper noise while maintaining the edge after the segmentation process was completed. The detail process was explained in [30].

\section{Results and Discussion}

The images of ALL and AML - which have undergone the dark-contrast algorithms that employed the TH cut-off point and $N T H g$ strain factor - are displayed in Fig. 7(c) and (d). With reference to the entirety of the sample, the pixel distribution of the leukocytes ranged from 40 to 100 . To increase the whiteness of the aforementioned TH area, the cut-off point and NTHg value was set as 90 and 255 respectively. The aim of this research was to eliminate irrelevant objects like erythrocytes and background pixels. Subsequently, the images' original colors were retained (Fig. 7(e) and (f)).

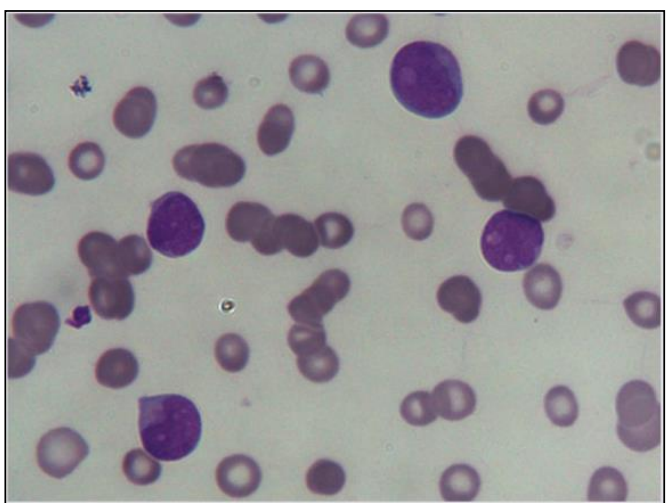

(a) AML image

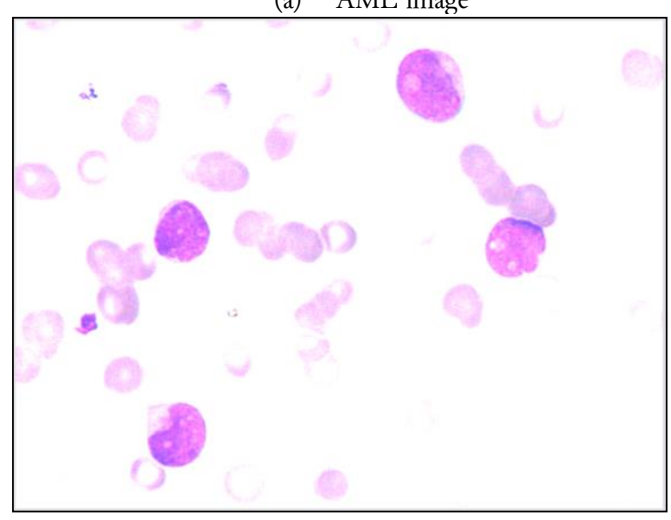

(c) AML image segmented after dark contrast process

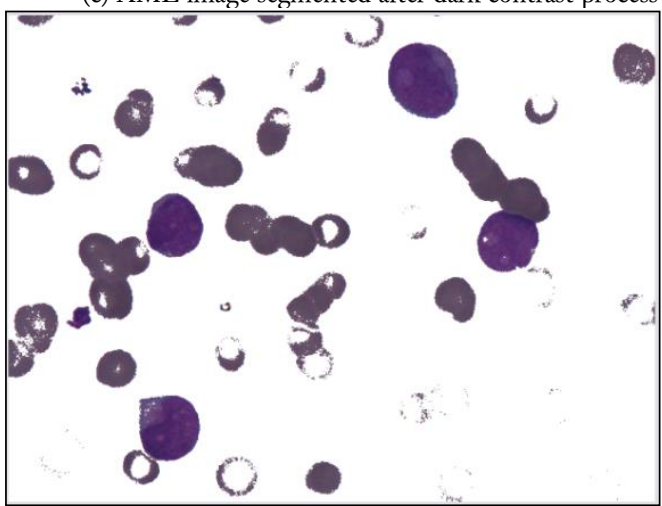

(e) Retrieve original AML image color

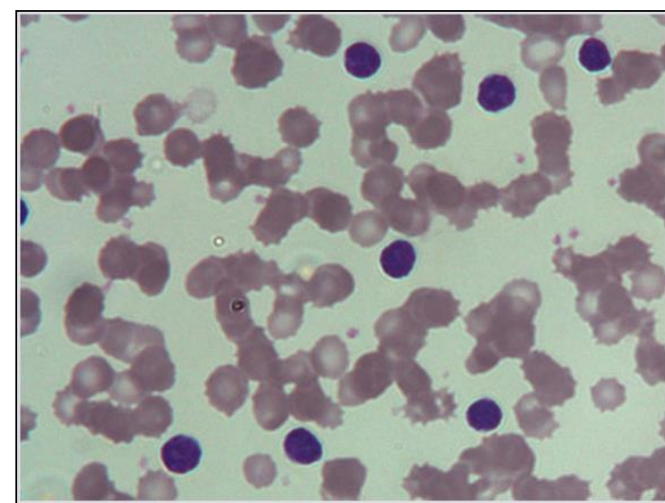

(b) ALL image

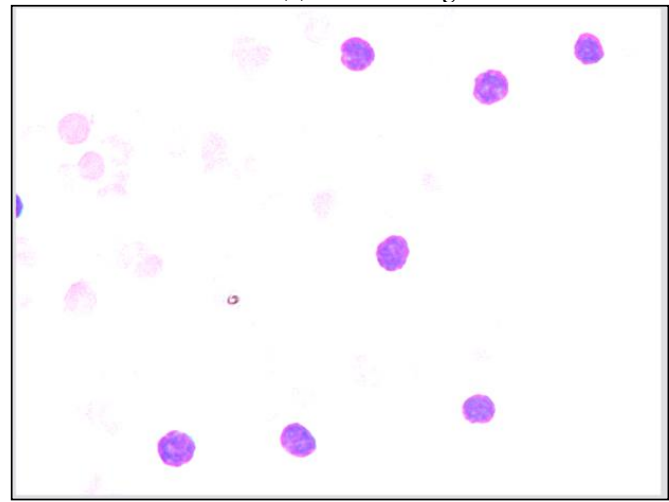

(d) ALL image segmented after dark contrast proces

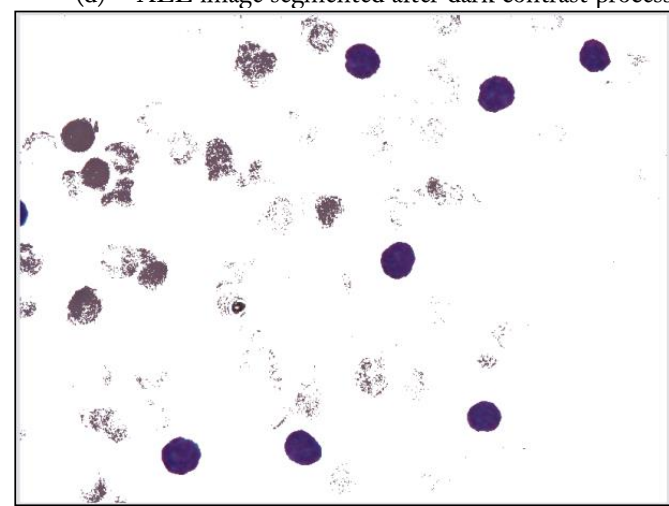

(f) Retrieve original ALL image color

Fig. 7. Result image AML and ALL from dark contrast method.

However, a salt and pepper noise still exist in the image of acute leukemia for example, in Fig. 7(e) and (f). To solve this problem, the median filter and SRGAE algorithm was used to eliminate and 
reduces the noise of salt and pepper as well as maintaining the edges. Fig. 8(a) and (b) shows the result image after applying a median filter with neighborhood $\mathrm{n}=7$, while Fig. 8(c) and (d) show the result image after applying SRGAE algorithm. The result shown the noise of salt and pepper remove better compare to Fig. $7(\mathrm{e})$ and (f).

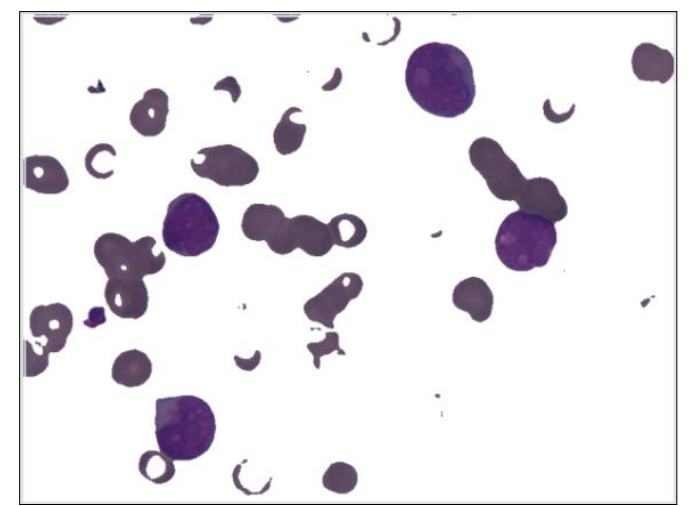

(a) Original color AML image segmented with filter $\mathrm{n}=7$

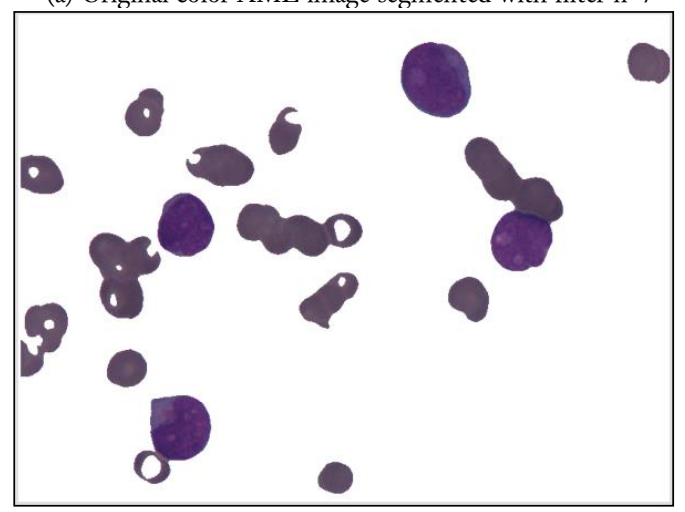

(c) SRGAE AML image segmented

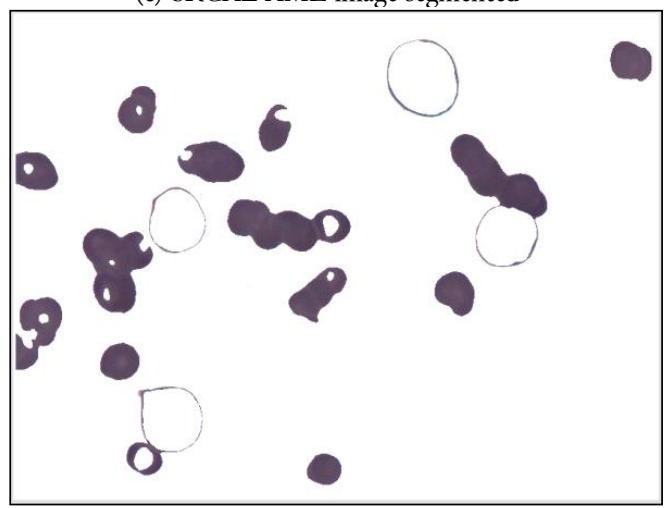

(e) Ghost AML image

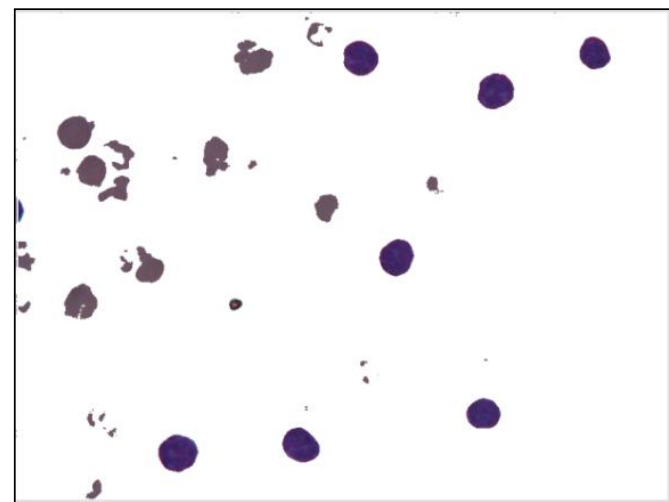

(b) Original color AML image segmented with filter $n=7$

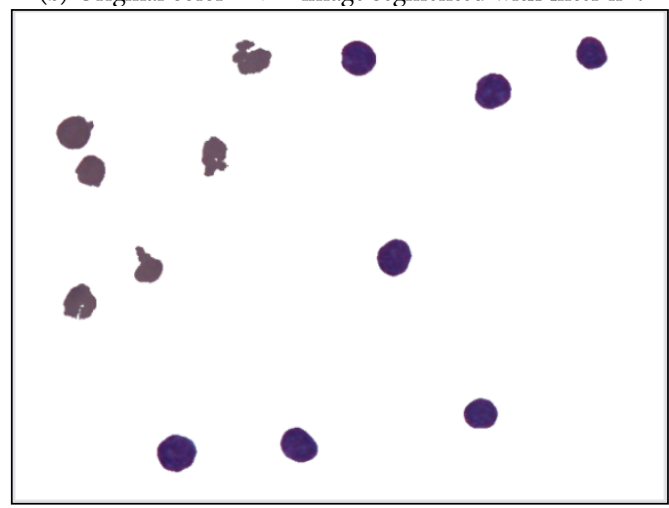

(d) SRGAE ALL image segmented

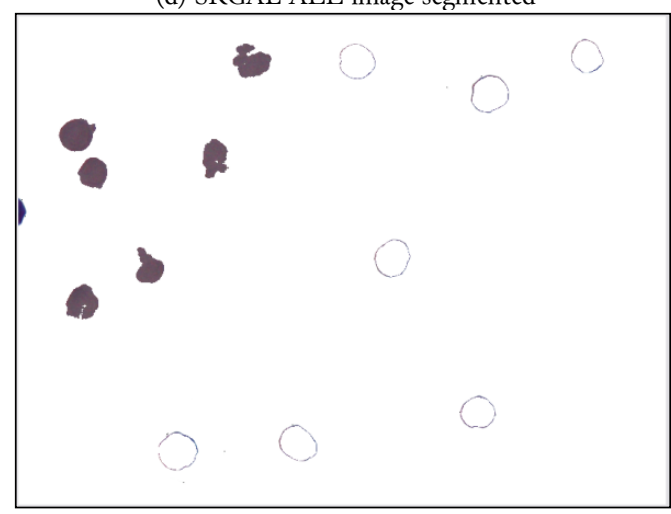

(f) Ghost ALL image

Fig. 8. Result segmented image after removing noise using filter $n=7$ and SRGAE algorithm.

To prove the effectiveness of DCA algorithm, the performance of DCA was calculated through overall accuracy (\%), sensitivity (\%) and specificity (\%). Table 1 compares the outcomes of the segmentation of 100 images (50 for ALL and AML respectively). The results analysis was observed based on the performance without the noise removal and after being combined with the noise removal. This study has focused on the accuracy value that has been obtained. Based on the segmentation performance of 100 acute leukemia images, the DCA method proposed with the noise removal algorithms has been proven better in producing segmented images in overall accuracy with $91.02 \%$ of average, $89.045 \%$ of AML image and $93.01 \%$ of ALL image, respectively. Similar with specificity, the result improve better with $91.57 \%$ for average, $89.30 \%$ for AML image and $93.84 \%$ for ALL image, respectively. However, the sensitivity value decreased after the noise removal process due to over-segmentation and inability to process fine input from noise. 
Table 1. Comparison performance of DCA.

\begin{tabular}{|c|c|c|c|c|}
\hline \multirow[b]{2}{*}{ Segmentation Process } & \multirow[b]{2}{*}{ Performance } & \multicolumn{3}{|c|}{ Image } \\
\hline & & $\begin{array}{c}\text { AML image } \\
(50)\end{array}$ & $\begin{array}{c}\text { ALL image } \\
(50)\end{array}$ & $\begin{array}{c}\text { Average } \\
(100)\end{array}$ \\
\hline \multirow[t]{3}{*}{ DCA without noise removal } & Overall accuracy (\%) & 87.34 & 89.77 & 88.56 \\
\hline & Sensitivity (\%) & 87.12 & 83.82 & 85.47 \\
\hline & Specificity (\%) & 87.39 & 90.24 & 88.82 \\
\hline \multirow[t]{3}{*}{ DCA with noise removal } & Overall accuracy (\%) & 89.04 & 93.01 & 91.02 \\
\hline & Sensitivity (\%) & 85.43 & 81.93 & 83.68 \\
\hline & Specificity (\%) & 89.30 & 93.84 & 91.57 \\
\hline
\end{tabular}

\section{Conclusion}

We have proposed a noise-removal DCA procedure which can enhance the segmentation of the images of acute lymphoblastic leukemia (ALL) and acute myeloid leukemia (AML). The result proves that the contrast method is important before proceeding to the leukemia screening in order to avoid misdiagnosis. Therefore, these medical images which are of higher clarity and cleanliness will facilitate as well as improve the disease-screening processes by the healthcare professionals.

\section{Acknowledgment}

We wish to thank the Malaysian Ministry of Higher Education as well as Universiti Utara Malaysia (UUM) under SO Code 13849 for their support of this study. Our heartfelt gratitude also goes to the leukemia research group of Universiti Malaysia Perlis (UniMAP) for realising this work. Special thanks to Hospital Universiti Sains Malaysia (HUSM) for their provision of the blood specimens.

\section{References}

[1] N. H. Harun, M. Y. Mashor, and R. Hassan, "Automated blasts segmentation techniques based on clustering algorithm for acute leukaemia blood samples," J. Adv. Comput. Sci. Technol. Res., vol. 1, pp. 96109, 2011, available at : http://www.sign-ific-ance.co.uk/index.php/JACSTR/article/view/375.

[2] S. Mishra, B. Majhi, and P. K. Sa, "A survey on automated diagnosis on the detection of Leukemia: A hematological disorder," in Recent Advances in Information Tecbnology (RAIT), 2016 3rd International Conference on, 2016, pp. 460-466, doi: https://doi.org/10.1109/RAIT.2016.7507945.

[3] H. G. Kaganami and Z. Beiji, "Region-Based Segmentation versus Edge Detection," in 2009 Fifth International Conference on Intelligent Information Hiding and Multimedia Signal Processing, 2009, pp. 12171221, doi: https://doi.org/10.1109/IIH-MSP.2009.13.

[4] I. Karoui, R. Fablet, J. M. Boucher, and J. M. Augustin, "Unsupervised region-based image segmentation using texture statistics and level-set methods," in 2007 IEEE International Symposium on Intelligent Signal Processing, 2007, pp. 1-5, doi: https://doi.org/10.1109/WISP.2007.4447617.

[5] Y. Zhou, S. Jiang, and M. Yin, "A Region-Based Image Segmentation Method with Mean-Shift Clustering Algorithm," in 2008 Fifth International Conference on Fuzzy Systems and Knowledge Discovery, 2008, pp. $366-$ 370, doi: https://doi.org/10.1109/FSKD.2008.363.

[6] A. M. Mendonca and A. Campilho, "Segmentation of retinal blood vessels by combining the detection of centerlines and morphological reconstruction," IEEE Trans. Med. Imaging, vol. 25, no. 9, pp. 1200-1213, Sep. 2006, doi: https://doi.org/10.1109/TMI.2006.879955.

[7] J. V. B. Soares, J. J. G. Leandro, R. M. Cesar, H. F. Jelinek, and M. J. Cree, "Retinal vessel segmentation using the 2-D Gabor wavelet and supervised classification," IEEE Trans. Med. Imaging, vol. 25, no. 9, pp. 1214-1222, Sep. 2006, doi: https://doi.org/10.1109/TMI.2006.879967.

[8] J. Staal, M. D. Abramoff, M. Niemeijer, M. A. Viergever, and B. van Ginneken, "Ridge-Based Vessel Segmentation in Color Images of the Retina," IEEE Trans. Med. Imaging, vol. 23, no. 4, pp. 501-509, Apr. 2004, doi: https://doi.org/10.1109/TMI.2004.825627.

[9] S. Lakshmi, D. V Sankaranarayanan, and others, "A study of edge detection techniques for segmentation computing approaches," IJCA Spec. Issue "Computer Aided Soft Comput. Tech. Imaging Biomed. Appl. CASCT, 
pp. 35-40, 2010, available at : http://citeseerx.ist.psu.edu/viewdoc/download?doi=10.1.1.206.3030\&rep= rep1\&type=pdf.

[10] M. Sharif, S. Mohsin, M. Y. Javed, and M. A. Ali, "Single Image Face Recognition Using Laplacian of Gaussian and Discrete Cosine Transforms.," Int. Arab J. Inf. Technol., vol. 9, no. 6, pp. 562-570, 2012, available at : https://pdfs.semanticscholar.org/03df/e517766a2cb914070c438a9396fb556158e8.pdf.

[11] M. Sharif, M. A. Ali, M. Raza, and S. Mohsin, "Face recognition using edge information and DCT," Sindh Univ. Res. Journal-SURJ (Science Ser., vol. 43, no. 2, 2015, available at : Google Scholar.

[12] S. Yuheng and Y. Hao, "Image Segmentation Algorithms Overview," arXiv Prepr. arXiv1707.02051, 2017, available at : https://arxiv.org/abs/1707.02051.

[13] S. Li, X. Kang, L. Fang, J. Hu, and H. Yin, "Pixel-level image fusion: A survey of the state of the art," Inf. Fusion, vol. 33, pp. 100-112, Jan. 2017, doi: https://doi.org/10.1016/j.inffus.2016.05.004.

[14] N. Dhanachandra, K. Manglem, and Y. J. Chanu, "Image Segmentation Using K -means Clustering Algorithm and Subtractive Clustering Algorithm," Procedia Comput. Sci., vol. 54, pp. 764-771, 2015, doi: https://doi.org/10.1016/j.procs.2015.06.090.

[15] N. M. Zaitoun and M. J. Aqel, "Survey on Image Segmentation Techniques," Procedia Comput. Sci., vol. 65, pp. 797-806, 2015, doi: https://doi.org/10.1016/j.procs.2015.09.027.

[16] Hongmin Cai, Zhong Yang, Xinhua Cao, Weiming Xia, and Xiaoyin Xu, "A New Iterative Triclass Thresholding Technique in Image Segmentation," IEEE Trans. Image Process., vol. 23, no. 3, pp. 10381046, Mar. 2014, doi: https://doi.org/10.1109/TIP.2014.2298981.

[17] N. Senthilkumaran and S. Vaithegi, "Image segmentation by using thresholding techniques for medical images," Comput. Sci. Eng. An Int. J., vol. 6, no. 1, pp. 1-13, 2016, available at : Google Scholar.

[18] S. Zhou, J. Wang, S. Zhang, Y. Liang, and Y. Gong, "Active contour model based on local and global intensity information for medical image segmentation," Neurocomputing, vol. 186, pp. 107-118, Apr. 2016, doi: https://doi.org/10.1016/j.neucom.2015.12.073.

[19] P. Ghosh, M. Mitchell, J. A. Tanyi, and A. Y. Hung, "Incorporating priors for medical image segmentation using a genetic algorithm," Neurocomputing, vol. 195, pp. 181-194, Jun. 2016, doi: https://doi.org/10.1016/j.neucom.2015.09.123.

[20] H. Gao, L. Dou, W. Chen, and G. Xie, "The applications of image segmentation techniques in medical CT images," in Proceedings of the 30th Chinese Control Conference, 2011, pp. 3296-3299, available at: https://ieeexplore.ieee.org/document/6001546.

[21] B. M. Ghafour, N. H. Salman, and G. M. Hadi, "Medical Image Segmentation Based on Edge Detection Techniques," J. Adv. Comput. Sci. Technol. Res., vol. 5, no. 1, pp. 16-27, 2015, available at : http://www.signific-ance.co.uk/index.php/JACSTR/article/download/1042/1009.

[22] A. N. A. Salihah, M. Y. Mashor, N. H. Harun, A. A. Abdullah, and H. Rosline, "Improving colour image segmentation on acute myelogenous leukaemia images using contrast enhancement techniques," in Biomedical Engineering and Sciences (IECBES), 2010 IEEE EMBS Conference on, 2010, pp. 246-251, doi: https://doi.org/10.1109/IECBES.2010.5742237.

[23] N. R. Mokhtar et al., "Image Enhancement Techniques Using Local, Global, Bright, Dark and Partial Contrast Stretching For Acute Leukemia Images," in Proceedings of the World Congress on Engineering 2009, 2009, vol. 1, available at : http://www.iaeng.org/publication/WCE2009/WCE2009_pp807-812.pdf.

[24] E. U. Francis, M. Y. Mashor, A. A. Azamimi, and H. Roseline, "Image enhancement technique using partial contrast stretching on abnormal Bone Marrow images for Leukemia screening," in International Postgraduate Conference On Engineering (IPCE 2010), 2010, available at : http://dspace.unimap.edu.my/xmlui/handle/ $123456789 / 21637$.

[25] M. Zhou, K. Jin, S. Wang, J. Ye, and D. Qian, "Color retinal image enhancement based on luminosity and contrast adjustment," IEEE Trans. Biomed. Eng., vol. 65, no. 3, pp. 521-527, 2018, doi: https://doi.org/10.1109/TBME.2017.2700627. 
[26] N. J. Willett, T. Thote, M. Hart, S. Moran, R. E. Guldberg, and R. V. Kamath, "Quantitative pre-clinical screening of therapeutics for joint diseases using contrast enhanced micro-computed tomography," Osteoarthr. Cartil., vol. 24, no. 9, pp. 1604-1612, Sep. 2016, doi: https://doi.org/10.1016/j.joca.2016.04.021.

[27] J. Khan, A. S. Malik, N. Kamel, S. C. Dass, and A. M. Affandi, "Contrast Enhancement of Color Images with Color Morphing Approach,” World Acad. Sci. Eng. Technol. Int. J. Comput. Electr. Autom. Control Inf. Eng., vol. 10, no. 2, pp. 437-440, 2017, available at: https://waset.org/publications/10006937/contrastenhancement-of-color-images-with-color-morphing-approach.

[28] E. Fröhlich, R. Muller, X.-W. Cui, D. Schreiber-Dietrich, and C. F. Dietrich, "Dynamic ContrastEnhanced Ultrasound for Quantification of Tissue Perfusion," J. Ultrasound Med., vol. 34, no. 2, pp. 179196, 2015, doi: https://doi.org/10.7863/ultra.34.2.179.

[29] A. R. Weeks, Fundamentals of electronic image processing. SPIE Optical Engineering Press Bellingham, 1996, available at: Google Scholar.

[30] N. H. Harun, M. Y. Mashor, L. H. Nee, and R. Hassan, "Automated white blood cells counting system for acute leukemia based on blood images," J. Teknol., vol. 78, no. 6-4, pp. 91-98, 2016, doi: https://doi.org/10.11113/jt.v78.8982. 